\title{
UNE NOUVELLE ESPÈCE D'EURYTREMA, E. TONKINENSE N. SP. PARASITE DU PANCRÉAS DES BOVIDÉS
}

\author{
Par H. GALLJARD et D. V. NGU
}

$\mathrm{Au}$ cours de nos recherches sur le rôle pathogène et l'évolution d'Eurytrema puncreaticum, parasite que nous rencontrons dans 50 p. ıoo des pancréas de bovins abattus à Hanoï, nous avons trouvé, chez deux de ces animaux, originaires de la province de Thanh-Hoa, des spécimens qui nous ont frappés par leur taille anormalement grande et leur coloration plus foncée.

L'infestation était intense et le fait que tous les spécimens étaient absolument identiques nous a fait penser qu'il s'agissait d'une espèce nouvelle. Une étude plus approfondie a confirmé cette opinion. Nous proposons de donner à cette espèce le nom d'Eurytrema tonkinense.

Cette espèce mesure $\mathrm{I} 2 \mathrm{~mm} .3$ à $\mathrm{I} 7 \mathrm{~mm} .8$ de long, sur $6 \mathrm{~mm} .2$ à $7 \mathrm{~mm} .5$ de large (spécimens étalés et fixés). Sa couleur est rouge. Le corps est foliacé, obtus en avant, arrondi en arrière. Les ventouses sont circulaires, la ventouse buccale a un diamètre de $2 \mathrm{~mm} .7$ à $2 \mathrm{~mm}$. 9, la ventouse ventrale a un diamètre de $\mathrm{I} \mathrm{mm}$. 8 à $2 \mathrm{~mm}$. I, elle est située vers le milieu du corps.

Les testicules, longs de 900 à $\mathrm{I} .350 \mu$, larges de 300 à $450 \mu$, sont placés symétriquement de chaque côté de la ventouse ventrale et ont leur extrémité antérieure à la hauteur du centre de cette dernière. Leur bord externe est profondément divisé en 3 ou 4 lobes très nets, tandis que leur bord interne est peu net et s'estompe.

La poche du cirre est allongée mais n'atteint pas le niveau de la ventouse ventrale; légèrement recourbée et dirigée d'arrière en avant et de dehors en dedans, elle aboutit au pore génital situé un peu en arrière de la bifurcation cæcale et sur la ligne médiane.

L'ovaire, situé en arrière de la ventouse ventrale, tantôt à droite, tantôt à gauche de la ligne médiane, mais toujours du mème côté que la poche du cirre, présente 3 à 5 digitations très nettes. Le canal de Laurer long et étroit décrit souvent une boucle près de son ANsiles de PARastrologir, T. XVIII, Nos 4-5-6. - 1941, p. 187-191. 


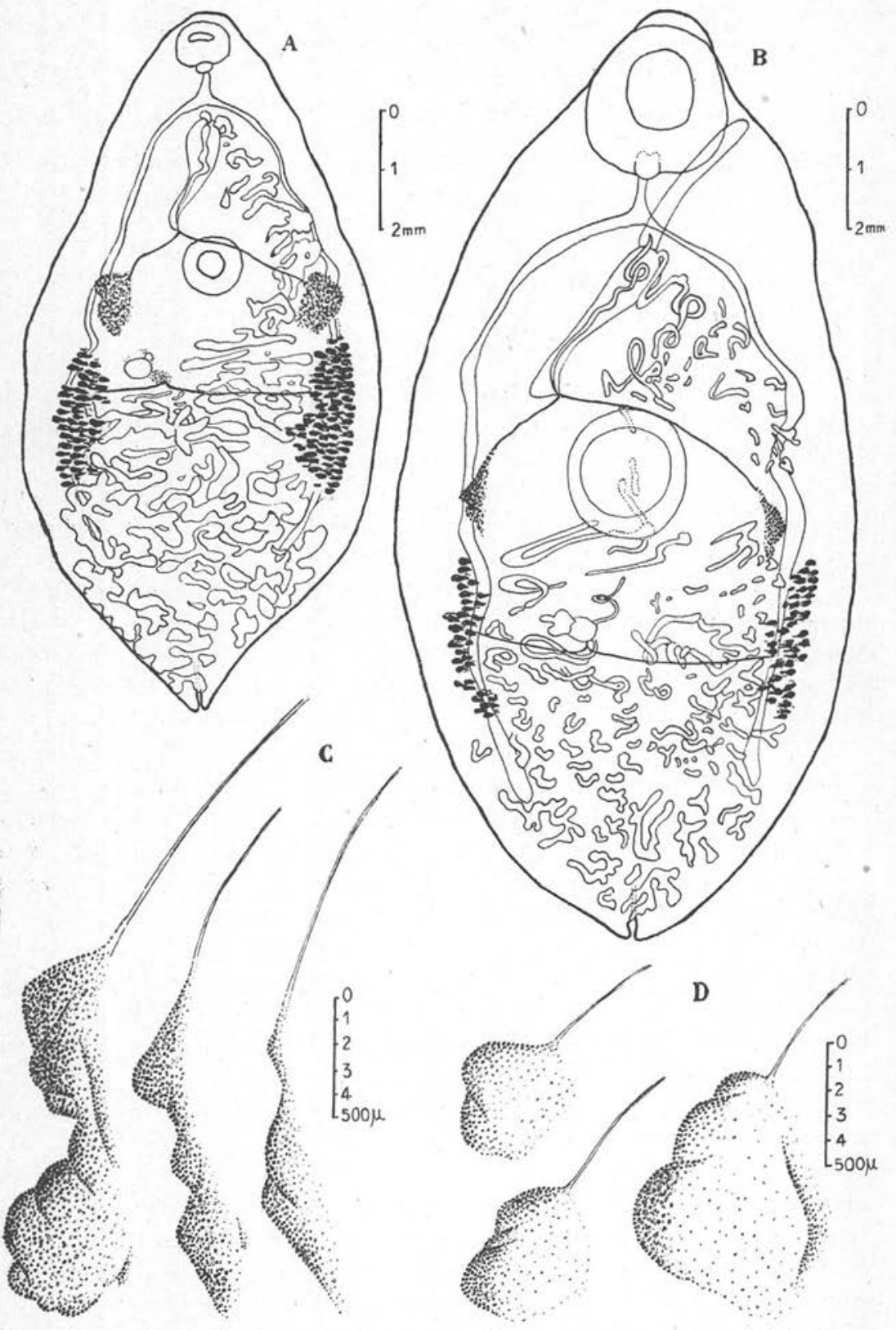

FIG. I. - A, Eurytrema pancreaticum; B, Eurytrema tonkinense; C, testicules d'E. tonkinense; D, testicules d'E. pancreaticum. 
extrémité libre, laquelle est paramédiane. Les vitellogènes sont allongés et situés latéralement vers la moitié postérieure du corps.

Ces caractères permettent facilement de distinguer $E$. tonkinense de $E$. pancreaticum. Alors que $E$. tonkinense a au moins $14 \mathrm{~mm}$.
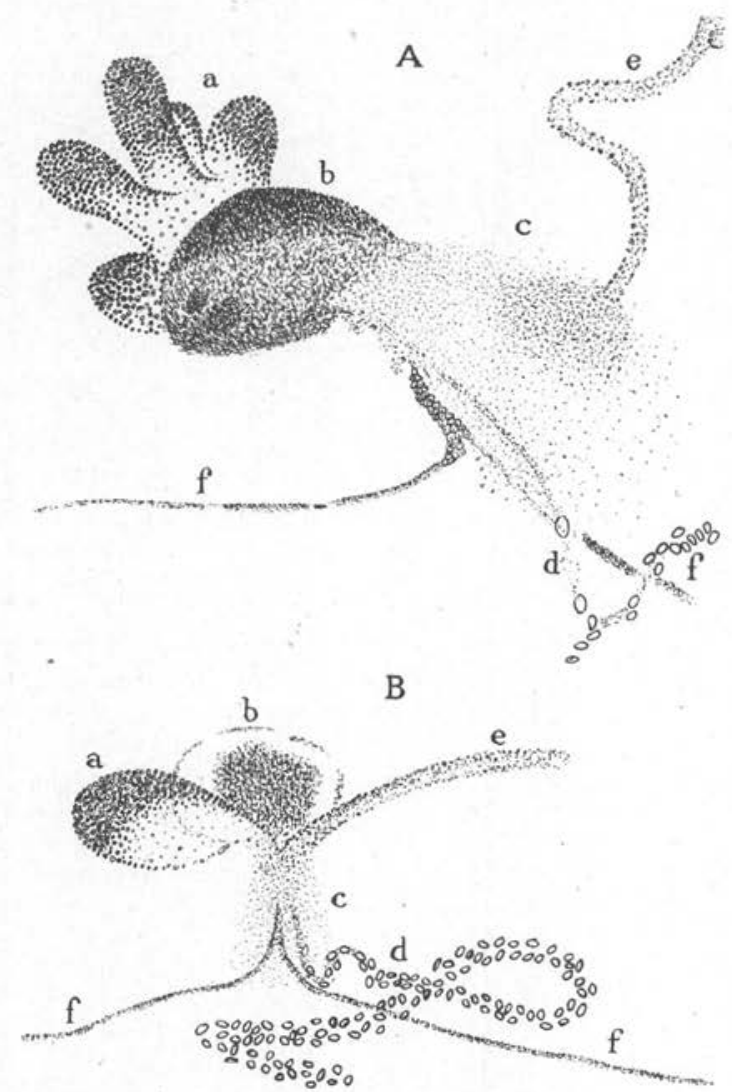

Fig. 2. - Appareil génital femelle d'Eurytrema tonkinense (A) et d'E. pancreaticum (B). - $a$, ovaire; $b$, réceptacle séminal ; $c$, glande coquillière; $d$, début de l'utérus; $e$, canal de Laurer; $f$, vitelloductes.

de longueur moyenne, $E$. pancreaticum a $9 \mathrm{~mm}$. et ne dépasse jamais I2 mm. parmi les milliers d'exemplaires que nous avons recueillis au Tonkin. Il est plus épais, sa couleur est donc d'un rouge sang plus foncé.

Mais le caractère distinctif le plus saillant est la position de la 


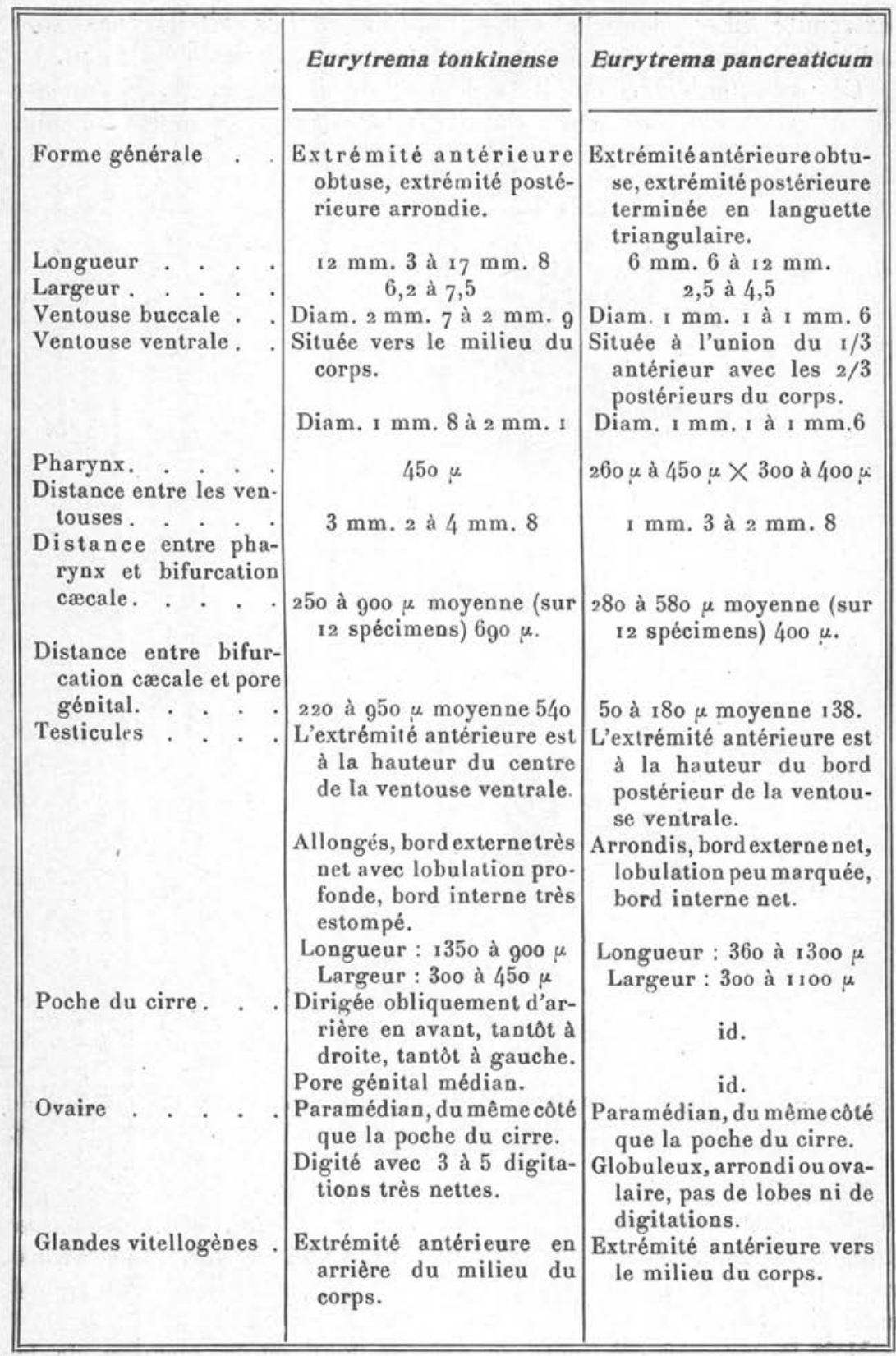


ventouse ventrale qui se trouve vers le milieu du corps, alors qu'elle est à l'union du tiers antérieur avec les deux tiers postérieurs chez $E$. pancreaticum. Enfin, les testicules sont allongés et profondément vallonnés sur leur bord externe, l'ovaire est nettement divisé alors que chez $E$. pancreaticum, ovaire et testicules sont arrondis ou vaguement lobés.

Ces caractères, résumés sur le tableau ci-joint, donnent à E. tonkinense une individualité certaine vis-à-vis d'E. pancreaticum tout d'abord, ainsi que d' $E$. colomaticum, trouvé également en Indochine.

Outre les différences d'hôtes et d'habitats, $E$. tonkinense se distingue certainement des Eurytrema parasites d'autres animaux, en particulier d'E. ovis (Marcos et Tubangui r $_{925} 5$ ) par la position des glandes vitellogènes qui sont dans le tiers moyen du corps et par la forme des testicules et des ovaires qui sont lobés dans cette dernière forme.

Laboratoire de Parasitologie de l'Ecole de Médecine de Hanoi. (Directeur: Prof. H.-G. Galliard). 